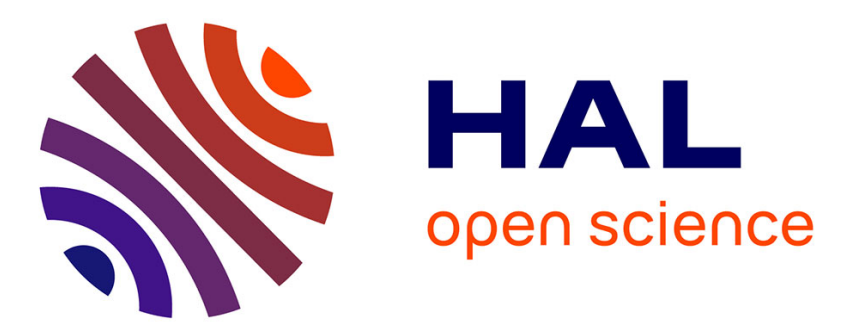

\title{
Experimental Application of Hybrid Fractional-Order Adaptive Cruise Control at Low Speed
}

Hassan Hosseinnia, Inés Tejado, Vicente Milanés, Jorge Villagrá, Blas Vinagre

\section{To cite this version:}

Hassan Hosseinnia, Inés Tejado, Vicente Milanés, Jorge Villagrá, Blas Vinagre. Experimental Application of Hybrid Fractional-Order Adaptive Cruise Control at Low Speed. IEEE Transactions on Control Systems Technology, 2014, pp.2329-2336. 10.1109/TCST.2014.2308837 . hal-01091158

\section{HAL Id: hal-01091158 https://hal.science/hal-01091158}

Submitted on 5 Dec 2014

HAL is a multi-disciplinary open access archive for the deposit and dissemination of scientific research documents, whether they are published or not. The documents may come from teaching and research institutions in France or abroad, or from public or private research centers.
L'archive ouverte pluridisciplinaire HAL, est destinée au dépôt et à la diffusion de documents scientifiques de niveau recherche, publiés ou non, émanant des établissements d'enseignement et de recherche français ou étrangers, des laboratoires publics ou privés. 


\title{
Experimental Application of Hybrid Fractional-Order Adaptive Cruise Control at Low Speed
}

\author{
S. Hassan Hosseinnia, Inés Tejado, Vicente Milanés, Jorge Villagrá, and Blas M. Vinagre
}

\begin{abstract}
This brief deals with the design and experimental application of a hybrid fractional adaptive cruise control (ACC) at low speeds. First, an improved fractional-order cruise control (CC) is presented for a commercial Citroën C3 prototype-which has automatic driving capabilities-at low speeds, which considers a hybrid model of the vehicle. The quadratic stability of the system is proved using a frequency domain method. Second, ACC maneuvers are implemented with two different distance policies using two cooperating vehiclesone manual, the leader, and the other, automatic-also at very low speeds. In these maneuvers, the objective is to maintain a desired interdistance between the leader and follower vehicles, i.e., to perform a distance control-with a proportional differential (PD) controller in this case-in which the previously designed fractional-order $\mathrm{CC}$ is used for the speed control. Simulation and experimental results, obtained in a real circuit, are given to demonstrate the effectiveness of the proposed control strategies.
\end{abstract}

Index Terms-Adaptive cruise control (ACC), fractional-order control (FOC), hybrid system and control, stability.

\section{INTRODUCTION}

$\mathbf{T}$ HE CONSIDERABLE increase in the number of vehicles for transportation of people or goods in recent decades have caused an increase in the number of road fatalities. Since more than $80 \%$ of road accidents are due to the human factor [1], it turns road transport into a suitable candidate to the application of autonomous or semiautonomous control systems to avoid-or reduce-driver errors.

During last years, significant advances have been carried out in this field. Although most of the commercial vehicles have included cameras or radars to detect pedestrians [2] or a leading vehicle [3], or ultrasound sensors for parking assistance [4], or even warning devices as head-up displays and audible signals, the last decision remains on the driver. Therefore, next step is to turn from warning to automatic devices for accident reduction or mitigation, which is an open field of research. Concerning vehicle's automation, one can

Manuscript received August 2, 2013; revised December 19, 2013; accepted February 23, 2014. Manuscript received in final form February 25, 2014. Date of publication April 1, 2014; date of current version October 15, 2014. This work was supported by the Spanish MICINN under Project TRA2008-06602C03-01 and Project TRA2008-06602-C03-02. Recommended by Associate Editor Y. Chen.

S. H. HosseinNia, I. Tejado, and B. M. Vinagre are with the Department of Electrical, Electronic, and Automation Engineering, Industrial Engineering School, University of Extremadura, Badajoz 06006, Spain (e-mail: hoseinnia@unex.es; itejbal@unex.es; bvinagre@unex.es).

V. Milanés and J. Villagrá are with the AUTOPIA Program, Centre for Automation and Robotics, Madrid 28500, Spain (e-mail: vicente.milanes@csic.es; jorge.villagra@csic.es).

Color versions of one or more of the figures in this paper are available online at http://ieeexplore.ieee.org.

Digital Object Identifier 10.1109/TCST.2014.2308837 distinguish between the lateral-associated to the steering wheel-or longitudinal-associated to the brake and throttle pedals - actions. The work presented in this brief focuses in the latter.

Automatic speed control-well known as cruise control (CC) in the literature-was one of the first autonomous systems implemented on a vehicle. It involves in regulating the action over the throttle pedal to try to follow a desired speed. Subsequent step was the inclusion of the brake pedal in the speed control system. Based on this inclusion and the use of radar system for detecting the leading vehicle, adaptive CC (ACC) systems were implemented for freeways driving [5]. Current research line in speed control is based on vehicle-to-vehicle ( $\mathrm{V} 2 \mathrm{~V})$ communications to reduce the distance between vehicles. These control systems, called cooperative ACC, have been experimentally tested with prototype vehicles [6]-[9].

Controlling the speed of a vehicle is a classic application of control system theory and, as a matter of fact, most of the commercial systems are based on proportional-integraldifferential (PID) controllers because of the proper vehicle's behavior versus their easy implementation. Although PID can achieve adequate results, advanced control techniques capable of improving their benefits are required in the automotive field. In this context, in the past few years, fractional-order PID controllers, i.e., the generalization of traditional PID to noninteger orders, are recognized to guarantee better closed-loop performance and robustness with respect to the classical controllers-refer to [10] and [11] for fundamentals and benefits of fractional-order control (FOC).

One of the key issues in longitudinal control is the commutation between the throttle and brake pedals due to the significant differences between the accelerating and braking vehicle dynamics. In this context, hybrid control-because of switching between different controllers - can be an accurate approach to achieve stability and provide an effective mechanism to deal with these highly complex systems by combining the advantages of different controllers [12]-[14]. Examples of hybrid controllers in the automotive field include applications for automated highway systems [15], motion planning [16], [17], collision avoidance [18], trajectory tracking [19], [20], and so on. Even though, research in hybrid control has been the object of an intense and productive effort in the recent years in the automotive field, from the best of our knowledge, this is the first time that benefits of both FOC and hybrid control are used for ACC maneuvers.

At this point, let us formulate the final aim of this brief as follows. Consider a number of vehicles driving in a common 
area at low speeds with $\mathrm{V} 2 \mathrm{~V}$ communications and a control station capable of communicating with all of them through a wireless network. The control station will be responsible for sending each car its specific target speed so as to avoid the possibility of collisions. The objective of this brief is to develop a capable and efficient advanced driver assistance system on the basis of FOC to allow the control station modify the speed of the cars to keep safety for all maneuvers in the area, which can be divided into two items: 1) remote CC maneuvers, which were addressed in [21] and 2) ACC via networks. In this brief, we will focus on the first step of this second item, more particularly, on the adaptation of the system developed in [21] to local ACC applications. With these premises, the purpose of this brief was threefold.

1) Design two fractional-order PI controllers to act over the throttle and brake pedals independently, so as to adapt the control designed in [21] for CC to ACC maneuvers.

2) Implement a hybrid control law for the commutation between the action over each pedal in a safe and robust way.

3) Validate the proposed hybrid control experimentally considering two different strategies for generating the safe interdistance between vehicles. It is worth mentioning that the idea was to compare two widely used rules with a classical PD controller, but not to design a fractionalorder one-it will be addressed in future works.

The rest of this brief is organized as follows. Section II briefly describes the follower vehicle and its dynamic longitudinal model obtained for this kind of maneuvers. Section III addresses CC maneuvers. In Section IV, ACC maneuvers and the interdistance policies considered for the experiments are described. Simulation and experimental results are given in Section $\mathrm{V}$ to validate the proposed hybrid fractional strategy for CC and ACC maneuvers. Finally, concluding remarks are included in Section VI.

\section{Automatic Vehicle}

This section briefly summarizes the modifications performed in the follower vehicle-an automatic convertible Citroën C3 Pluriel, whereas the leader is an electric Citroën Berlingo- to act autonomously on the throttle and brake pedals for the experimental CC and ACC maneuvers, as well as its dynamic longitudinal model when accelerating and braking at very low speeds. Both follower and leader vehicles belong to the AUTOPIA Program at the Center for Automation and Robotics (CAR).

\section{A. Description}

The vehicle control system for automatic driving, following the classical perception-reasoning-action paradigm [22], [23], is in charge of localizing as precisely and robustly as possible the vehicle. To that end, the following subsystems are embedded in the vehicle.

1) A double-frequency global positioning system (GPS) receiver running in real-time kinematic (RTK) carrier phase differential mode that supplies $2 \mathrm{~cm}$ of resolution positioning at a refresh rate of $5 \mathrm{~Hz}$.
2) A wireless local area network (IEEE 802.11) support, which allows the GPS to receive both positioning error corrections from its base station and vehicle and positioning information from the preceding vehicle.

3) An inertial measurement unit (IMU) Crossbow IMU 300CC placed close to the center of the vehicle to provide positioning information during GPS outages.

4) Car odometry supplied by a set of built-in sensors in the wheels, whose measurements can be read by accessing the controller area network bus of the vehicle.

5) An on-board computer, which requests values from each of the on-board sensors with which to compute the controller's input values.

Finally, the devices that make possible to act on the throttle and brake of the car are an electrohydraulic system, which is mounted in parallel with the original one, capable of injecting pressure into the car's antiblock braking system, and an analog card, which can send a signal to the car's internal engine computer to demand acceleration or deceleration. More details can be found in [24].

\section{B. Dynamic Longitudinal Model}

To design the controllers for $\mathrm{CC}$ and ACC maneuvers at very low speeds, a model of the vehicle was obtained experimentally when accelerating and braking. It is worth mentioning that obtaining the exact vehicle longitudinal dynamics is not required in this application because of the kind of experimental maneuvers to be performed. Thus, simple linear models were considered-similar models have been also used in [25] and [26]. On the one hand, the vehicle speed when accelerating was simplified as

$$
G_{1}(s) \simeq \frac{4.39}{s+0.1746} .
$$

On the other hand, the vehicle dynamics when braking can be given by an uncertain first-order transfer function that depends on the voltage applied to the brake pedal [24]

$$
G_{2}(s) \simeq \frac{1}{\tau s+1}
$$

where the time constant varies in the interval $\tau \in[1.6,3.1] \mathrm{s}-$ the nominal value of $\tau$ was considered as 2.25 . The validation of these models can be found in [21], [27], and [28].

\section{CRUise CONTROL}

This section presents the hybrid CC of the vehicle at low speeds based on the above-mentioned different dynamics. The design of the fractional-order controllers for the throttle and the brake is firstly given and then, the hybrid modeling, control, and stability analysis.

\section{A. Design of the Fractional-Order Controllers}

The most important mechanical and practical requirement of the vehicle to consider during the design process is to obtain a smooth vehicle's response so as to guarantee its acceleration to be less than the well-known comfort acceleration, i.e., less than $2 \mathrm{~m} / \mathrm{s}^{2}$. 
In [21], [27], and [29], some classical and fractional-order PI controllers were designed for CC maneuvers. In this brief, the fractional-order PI controller designed in [27] will be used for the throttle action-it was designed to control the throttle and brake pedals, but neglecting the dynamics during braking-whereas the brake will be controlled by a robust fractional-order PI due to the system uncertainty described previously. The motivation of improving that design by considering a hybrid model of the vehicle mainly arises from its application to ACC maneuvers, in which commutation between the pedals plays a key role for the success of the whole control.

Consider a fractional-order PI controller of the form

$$
C(s)=k_{p}+\frac{k_{i}}{s^{\alpha}} .
$$

Specifications related to phase margin, gain crossover frequency and output disturbance rejection are going to be considered. Let assume that the gain and phase crossover frequency of the open-loop system are given by $\omega_{\mathrm{gc}}$ and $\omega_{\mathrm{pc}}$, the phase and gain margins are denoted by $\phi_{m}$ and $M_{g}$, and the output disturbance rejection is defined by a desired value of a sensitivity function $S(s)$ for a desired frequencies range. The three specifications to be fulfilled to achieve stability and robustness are the following.

1) Phase margin specification

$$
\begin{aligned}
& \arg \left(C\left(j \omega_{\mathrm{gc}}\right) G\left(j \omega_{\mathrm{gc}}\right)\right)=-\pi+\phi_{m} \\
& \arg \left(C\left(j \omega_{\mathrm{pc}}\right) G\left(j \omega_{\mathrm{pc}}\right)\right)=-\pi .
\end{aligned}
$$

2) Gain crossover frequency specification

$$
\begin{aligned}
\left|C\left(j \omega_{\mathrm{gc}}\right) G\left(j \omega_{\mathrm{gc}}\right)\right| & =1 \\
\left|C\left(j \omega_{\mathrm{pc}}\right) G\left(j \omega_{\mathrm{pc}}\right)\right|_{\mathrm{dB}} & =1 / M_{g} .
\end{aligned}
$$

3) Output disturbance rejection

$$
\left|\frac{1}{1+C(j \omega) G(j \omega)}\right|_{\mathrm{dB}} \leq-20 \mathrm{~dB}, \quad \omega \leq \omega_{s} .
$$

To tune the fractional-order PI controller (3) for the throttle, the set of (4)-(6)-(8) was solved with the MATLAB function fsolve for the following specifications: 1) $\phi_{m}=90^{\circ}$;2) $\omega_{\mathrm{gc}}=$ $0.45 \mathrm{rad} / \mathrm{s}$; and 3) $\omega_{s}=0.035 \mathrm{rad} / \mathrm{s}$. The controller parameters were: 1) $k_{p}=0.09$; 2) $k_{i}=0.025$; and 3) $\alpha=0.8$-the full design of this controller can be found in [27].

With respect to the control of the brake, a fractional-order PI controller robust to variations in the system time constant was required. Since the controlled system phase never crosses $-\pi \mathrm{rad}$, and in accordance with the idea proposed in [30], the following specifications are considered instead of (5) and (7):

$$
\begin{aligned}
\arg \left(C\left(j \omega_{m}\right) G\left(j \omega_{m}\right)\right) & =p_{m} \\
\left|C\left(j \omega_{m}\right) G\left(j \omega_{m}\right)\right|_{\mathrm{dB}} & =1 / M_{m} .
\end{aligned}
$$

Thus, (9) and (10) are used to find the frequency $\omega_{m}$ at the gain $M_{m}$ and the phase $p_{m}$. Therefore, defining $\theta=\alpha \pi / 2$, (4), (6), (9), and (10) turned into the following set of four nonlinear

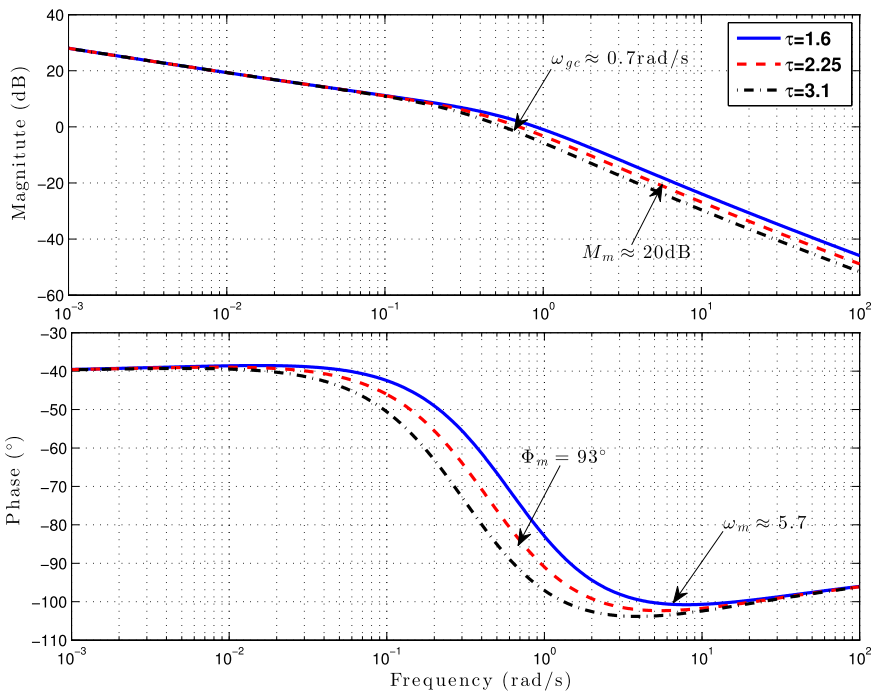

Fig. 1. Bode diagrams of the vehicle controlled by the fractional-order PI with different values of the time constant $\tau$ for the brake.

equations with four unknown variables $-k_{p}, k_{i}, \alpha$, and $\omega_{m}$ :

$$
\tan ^{-1}\left(\frac{k_{p} \omega_{\mathrm{gc}}^{\alpha} \sin \theta}{k_{i}+k_{p} \omega_{\mathrm{gc}}^{\alpha} \cos \theta}\right)-\tan ^{-1}\left(\tau \omega_{\mathrm{gc}}\right)+\pi-\theta-\phi_{m}=0
$$

$$
\tan ^{-1}\left(\frac{k_{p} \omega_{m}^{\alpha} \sin \theta}{k_{i}+k_{p} \omega_{m}^{\alpha} \cos \theta}\right)-\tan ^{-1}\left(\tau \omega_{m}\right)-\theta-p_{m}=0
$$

$$
20 \log \left(\frac{\sqrt{\left(k_{i}+k_{p} \omega_{\mathrm{gc}}^{\alpha} \cos \theta\right)^{2}+\left(k_{p} \omega_{\mathrm{gc}}^{\alpha} \sin \theta\right)^{2}}}{\omega_{\mathrm{gc}}^{\alpha} \sqrt{\left(\tau \omega_{\mathrm{gc}}\right)^{2}+1}}\right)=0
$$

$$
20 \log \left(\frac{\sqrt{\left(k_{i}+k_{p} \omega_{m}^{\alpha} \cos \theta\right)^{2}+\left(k_{p} \omega_{m}^{\alpha} \sin \theta\right)^{2}}}{\omega_{m}^{\alpha} \sqrt{\left(\tau \omega_{m}\right)^{2}+1}}\right)-\frac{1}{M_{m}}=0 .
$$

In this case, the MATLAB function fmincon was used to reach out its solution, which finds the constrained minimum of a function of several variables. Actually, (13) was considered as the main function to optimize with (11), (12), and (14) as constraints. Considering $\phi_{m}=90^{\circ}, p_{m}=-100^{\circ}$, $\omega_{\mathrm{gc}}=0.7 \mathrm{rad} / \mathrm{s}$, and $M_{m}=20 \mathrm{~dB}$ as specifications, the parameters obtained for the brake controller were $k_{p}=0.7$, $k_{i}=1.1, \alpha=0.45$, whereas $\omega_{m}=5.7 \mathrm{rad} / \mathrm{s}$. This controller was tuned to be robust for the above-mentioned interval for $\tau$. Fig. 1 shows the Bode diagrams of the vehicle when braking with the designed $\mathrm{PI}^{\alpha}$ controller. It can be observed that $\omega_{\mathrm{gc}}=$ $0.7 \mathrm{rad} / \mathrm{s}$ and $\phi_{m}=93^{\circ}$, which fulfill the design specifications with robustness to variations of system time constant $\tau$.

\section{B. Hybrid Control}

Let us describe the system and the controllers by their transfer functions $G_{q}(s)=K_{q} / s+T_{q}$ and $C_{q}(s)=k_{p_{q}}+$ $k_{i_{q}} / s^{\alpha_{q}}$, where $q=\{1,2\}$ refers to the throttle and brake actions, respectively, and with the parameters shown in Table I. 
TABLE I

SYSTEM AND CONTROLLER PARAMETER

\begin{tabular}{|l|c|c|c|c|c|}
\hline & $K_{q}$ & $T_{q}$ & $k_{p_{q}}$ & $k_{i_{q}}$ & $\alpha_{q}$ \\
\hline Throttle $(q=1)$ & 4.39 & 0.175 & 0.09 & 0.025 & 0.8 \\
\hline Brake $(q=2)$ & $1 / \tau$ & $1 / \tau$ & 0.7 & 1.1 & 0.45 \\
\hline
\end{tabular}

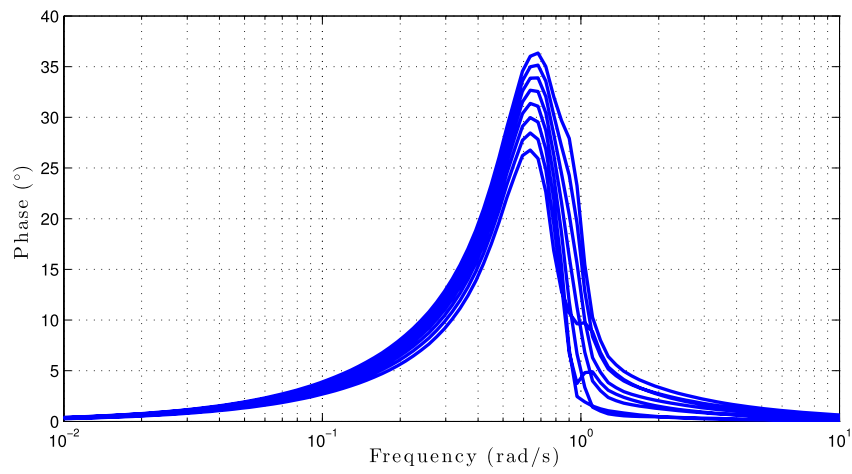

Fig. 2. Phase differences between the characteristic polynomials of the closed-loop system.

Thus, the closed-loop transfer function of the system can be written as

$$
\frac{Y(s)}{R(s)}=\frac{\gamma_{q} s^{\alpha_{q}}+\beta_{q}}{s^{\alpha_{q}+1}+\left(T_{q}+\gamma_{q}\right) s^{\alpha_{q}}+\beta_{q}}, \quad q=\{1,2\}
$$

where $\gamma_{q}=K_{q} k_{p_{q}}$ and $\beta_{q}=K_{q} k_{i_{q}}$.

To analyze the stability of the hybrid system, the frequency domain method proposed in [31] is going to be used. To this respect, the system has to be described as a switching system. Therefore, assuming $\alpha_{q}=N_{q} / D_{q}$, the switching system can be represented as

$$
D^{\frac{1}{M_{q}}} x=A_{q} x
$$

where $A_{q}, q=\{1,2\}$, are the switching subsystems and $M_{q}$ is lowest common multiple of the denominators of $D_{q}$ and $N_{q}$. Then, system (16) is quadratically stable if and only if

$$
\left|\arg \left(\operatorname{det}\left(\mathscr{A}_{1}-j \omega I\right)\right)-\arg \left(\operatorname{det}\left(\mathscr{A}_{2}-j \omega I\right)\right)\right|<\frac{\pi}{2} \quad \forall \omega
$$

where $\mathscr{A}_{q}=-\left(-A_{q}\right)^{1 / 2-M_{q}}, q=\{1,2\}$, and $I$ is identity matrix with proper dimension. The characteristic polynomials of the controlled system for the throttle and the brake are $d_{1}=s^{1.8}+0.57 s^{0.8}+0.11$ and $d_{2}=s^{1.45}+0.76 s^{0.45}+0.49$, respectively. Thus, the corresponding fractional-order system can be represented in state space by

$$
\begin{aligned}
D^{\frac{1}{5}} x & =A_{1} x=\left[\begin{array}{cccc}
O_{1,5} & -0.57 & O_{1,3} & -0.11 \\
& I_{9,9} & & O_{9,1}
\end{array}\right] x \\
D^{\frac{1}{20}} x & =A_{2} x=\left[\begin{array}{cccc}
O_{1,20} & -0.76 & O_{1,8} & -0.49 \\
& I_{29,29} & & O_{29,1}
\end{array}\right] x
\end{aligned}
$$

where $O_{l, m}$ and $I_{l, m}$ denote matrix of zeros and identity matrix with dimension of $l \times m$, respectively. Fig. 2 shows the graphic representation of condition (17) applied to this system. It can be observed that the maximum phase difference is $36.33^{\circ}$ independently of $\tau$-less than $90^{\circ}$ - which prove the stability of the controlled system considering the uncertainty in the brake dynamics.

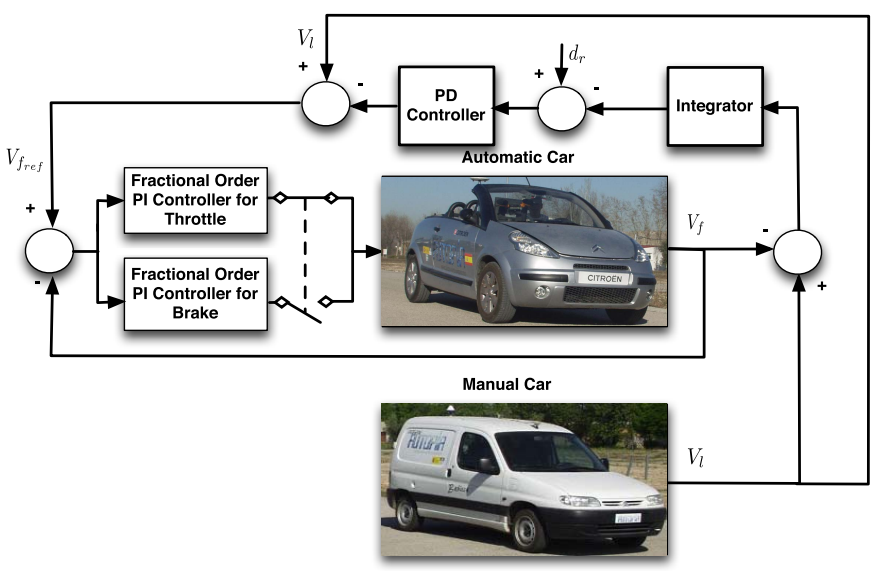

Fig. 3. Scheme of ACC maneuvers with two Citroën vehicles.

\section{ADAPTIVE CC}

This section addresses ACC maneuvers with two different distance policies considering two cooperating vehicles-one manual, the leader, and another automatic - at very low speeds (see a scheme in Fig. 3). The objective is to act the throttle and the brake of the automatic vehicle to track as precisely as possible both a desired distance between the two vehicles (interdistance) and a target relative velocity. Actually, a classical PD controller will be designed to perform the interdistance control, whereas the previously designed hybrid fractionalorder control will be used for the longitudinal control of the automatic vehicle. Thus, at least two control law regimes are needed: one for the desired velocity tracking (problem studied in Section III) and the other which tracks a desired following distance between the leader vehicle and a detected lead vehicle.

\section{A. Interdistance Policies}

In ACC, it is necessary to set the interdistance in a safe distance, which is called safe interdistance, $d_{r}$, and will be the reference distance for the control. Although different strategies have been proposed in the literature to obtain $d_{r}$, we will focus on the distance policies reported in [32] and [33] mainly due to their success.

In accordance with [32], $d_{r}$ has been calculated as the minimal distance to avoid a collision if the preceding vehicle were to act unpredictably

$$
d_{r}=h V+d_{c}+l_{v}
$$

which is known as constant-time headway policy, where $l_{v}$ is the vehicle length, $d_{c}$ is the minimal interdistance to avoid collision, and $V$ is vehicle velocity and $h$ is the constanttime headway, which is specified by the driver. No collision can occur if it is satisfied $h \geq 2 \gamma_{\max } / J_{\max }$, where $\gamma_{\max }$ and $J_{\max }$ are the maximum attainable vehicle's acceleration and the maximum driver desired jerk, respectively [34].

On the other hand, a safe interdistance policy is proposed in [33] in such a way control could be designed independently of the vehicle's model, permitting the additional control loop to only be responsible of the model-matching between the actual 


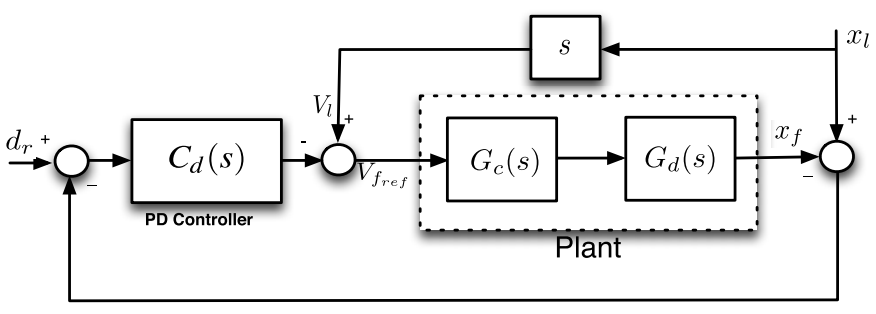

Fig. 4. Scheme of the closed-loop control of the automatic vehicle for ACC maneuvers.

system and the desired reference dynamics. The dynamic reference model will provide a reference interdistance less than the 2-s headway rule if the allowed maximum acceleration is high enough. In particular, the interdistance reference model describes the virtual dynamics of a vehicle, which is positioned at a reference distance $d_{r}$ from the leading vehicle as follows [33]:

$$
\dot{d}_{r}=c\left(d_{0}-d_{r}\right)^{2}+\dot{x}_{l}(t)-\left[c\left(d_{0}-d_{r}(0)\right)^{2}+\dot{x}_{f}(0)\right]
$$

where $d_{0}$ is the nominal safe interdistance, $c$ plays the role of a damping constant-from a nonlinear model-, $x_{l}$ is the position of the leading vehicle, and $\dot{x_{f}}$ is the velocity of the follower. Note that all $l$ and $f$ subscripts refer to leading and following vehicles, respectively.

It should be remarked that both interdistance policies (18) and (19) satisfy the following comfort and safety constraints: 1) $d_{r} \geqslant d_{c}$; 2) $\left|\dot{V}_{f}\right| \leqslant \gamma_{\max }$; and 3) $\left|\ddot{V}_{f}\right| \leqslant J_{\max }$. They are taken to represent the worst case scenario in an emergency and limitations on the response of the traction and braking systems in the vehicle, as well as what is physiologically tolerable for the occupants.

\section{B. Design of the Interdistance Controller}

In this section, a classical PD controller is going to be designed to obtain the reference speed for the following vehicle and guarantee the tracking of $d_{r}$, which will be generated with the aforementioned policies.

A block diagram of the closed-loop control to be performed in the vehicle is shown in Fig. 4. The inner loop system can be expressed as $F(s)=C_{d}(s) G_{c}(s) G_{d}(s)$, where $C_{d}, G_{c}$, and $G_{d}$ denote the transfer functions of PD controller, the closed-loop longitudinal control and a traditional integrator, respectively, i.e., $C_{d}(s)=k_{p}+k_{d} s, G_{c}(s)=$ $C_{q}(s) G_{q}(s) / 1+C_{q}(s) G_{q}(s)$, and $G_{d}(s)=1 / s$.

To design a unique PD for the two inner-loop systems because of the brake and throttle dynamics, the system with lower phase margin was considered: the dynamics when throttle is active. Considering the following design specifications for the inner loop:

$$
\begin{aligned}
\arg \left(F\left(j \omega_{\mathrm{gc}}\right)\right) & =-\pi+\phi_{m} \\
\left|F\left(j \omega_{\mathrm{gc}}\right)\right| & =0 \mathrm{~dB}
\end{aligned}
$$

with $\phi_{m}>80^{\circ}$ and $0.6<\omega_{\mathrm{gc}}<1 \mathrm{rad} / \mathrm{s}$, the parameters obtained for the PD controller were: $k_{p}=0.7$ and $k_{d}=1.2$.

\section{Simulation And ExPERIMENTAl Results}

The real experiments were carried out on the real vehicle in the CARs private driving circuit, which was designed with scientific purposes, so only experimental vehicles were driven in this area. Two vehicles were used for the experimental phase: a fully automated vehicle and a manually driven one. As commented, the former is a convertible Citroën C3 Pluriel and is equipped with automatic driving capabilities with hardware modifications made to the throttle and the brake pedal actions. The latter vehicle is an electric Citroën Berlingo van also equipped with automatic driving capabilities. For the purpose of this brief, it was driven by a human driver making the leading car's behavior as close to a real traffic situation as possible. Both vehicles were equipped with RTK-DGPS working at $5 \mathrm{~Hz}$ as the main sensor.

This section shows the goodness of the proposed fractional hybrid strategy through simulation and experimental results, grouped into CC and ACC maneuvers. First, the details of how to implement the fractional-order controllers digitally are given.

\section{A. Digital Implementation of Fractional Order Controllers}

Theoretically, a fractional-order controller is an infinitedimensional linear filter, and that all existing implementation schemes are based on finite-dimensional approximations. In practice, we use a digital method, specifically the indirect discretization method, which requires two steps: 1) obtaining a finite-dimensional continuous approximation for the integral part $s^{-\alpha}$ and 2) discretizing the resulting $s$-transfer function. In our case, to preserve the integral effect, $s^{-\alpha}$ was implemented as $s^{-\alpha}=s^{-1} s^{1-\alpha}$; actually, only the fractional part $P(s)=s^{1-\alpha}$ was approximated by the modified Oustaloup's method [10]. Thus, an integerorder transfer function that fits the frequency response of $P(s)$ in the range $\omega \in\left(10^{-3}, 10^{3}\right) \mathrm{rad} / \mathrm{s}$ was obtained with seven poles and seven zeros. Later, the discretization of this continuous approximation was carried out using the Tustin rule with a sampling period $T_{s}=0.2 \mathrm{~s}$-GPS sampling period. Considering both the throttle and the brake controllers, eight-order digital IIR filters of the form $C_{q}(z)=$ $k_{p_{q}}+k_{i_{q}}\left(2 / T_{s} 1-z^{-1} / 1+z^{-1}\right)^{-1} P_{q}(z)$ were obtained, where $P_{q}(z)=\sum_{k=0}^{7} b_{k} z^{-k} / 1+\sum_{k=1}^{7} a_{k} z^{-k}$ and with the coefficients shown in Table II.

\section{B. Results for CC Maneuvers}

The designed hybrid controller for CC maneuvers was tested by MATLAB/Simulink simulations and on the real automatic vehicle for different low velocity references between 5 and $20 \mathrm{~km} / \mathrm{h}$. In simulation, a random noise with zero mean and variance of 0.85 was added to the nominal value of $\tau$ to show the efficiency of the robust controller.

Fig. 5 shows the behavior of the vehicle when applying the designed fractional-order hybrid controller; more precisely, velocity tracking, acceleration, and normalized control action are included. In Fig. 5(a), the solid and dash-dotted lines refer to the experimental and simulated responses, whereas 
TABLE II

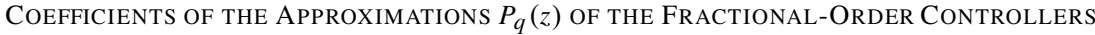

\begin{tabular}{|l|c|c|c|c|c|c|c|c|}
\hline & $b_{0}$ & $b_{1}$ & $b_{2}$ & $b_{3}$ & $b_{4}$ & $b_{5}$ & $b_{6}$ & $b_{7}$ \\
\hline Throttle $(q=1)$ & 0.157 & 0.133 & -0.439 & -0.366 & 0.406 & 0.334 & -0.124 & -0.101 \\
\hline Brake $(q=2)$ & 0.353 & 0.188 & -1.027 & -0.538 & 0.996 & 0.513 & -0.321 & -0.162 \\
\hline \hline & & $a_{1}$ & $a_{2}$ & $a_{3}$ & $a_{4}$ & $a_{5}$ & $a_{6}$ & $a_{7}$ \\
\hline Throttle $(q=1)$ & & -0.866 & -2.746 & 2.339 & 2.507 & -2.095 & -0.760 & 0.621 \\
\hline Brake $(q=2)$ & & -0.540 & -2.881 & 1.505 & 2.766 & -1.395 & -0.885 & 0.430 \\
\hline
\end{tabular}
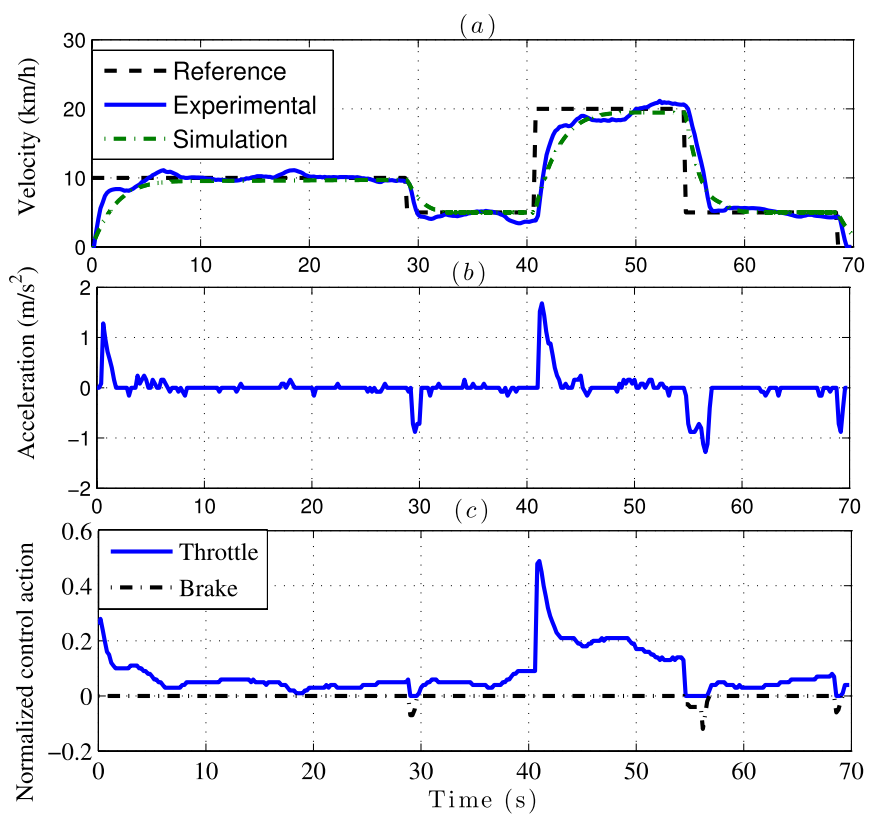

Fig. 5. CC results. (a) Velocity. (b) Acceleration. (c) Normalized control action.

the dotted lines is the velocity reference. First, it is worth mentioning that both the experimental and the simulated behaviors are quite similar, so the considered longitudinal dynamics of the vehicle is good enough for the maneuvers at low speeds. Furthermore, the simulated and experimental vehicle responses are stable and smooth and track the desired reference. In Fig. 5(c), both the throttle and the brake control inputs were normalized to the interval $[-1,1]$, where positive values mean throttle actions-solid line-and the negative, brake ones-dotted line. It can be observed that the acceleration and control action are met the desired intervals. One can also appreciate the soft action over vehicle's actuators obtaining a good comfort for car's occupants-this is reflected in the acceleration values.

To sum up, the fractional-order hybrid control may be useful for autonomous vehicles at low speeds to control both the brake and the throttle actions, specially due to its possibility of obtaining more adjustable time and frequency responses and allowing the fulfillment of more robust performances.

\section{Results for ACC Maneuvers}

To compare the control with the two interdistance policies in conditions as equal as possible, a predefined route was recorded. This route was first travelled over with the manually driven vehicle, and all the relevant variables to perform the control-position, speed and acceleration were stored.
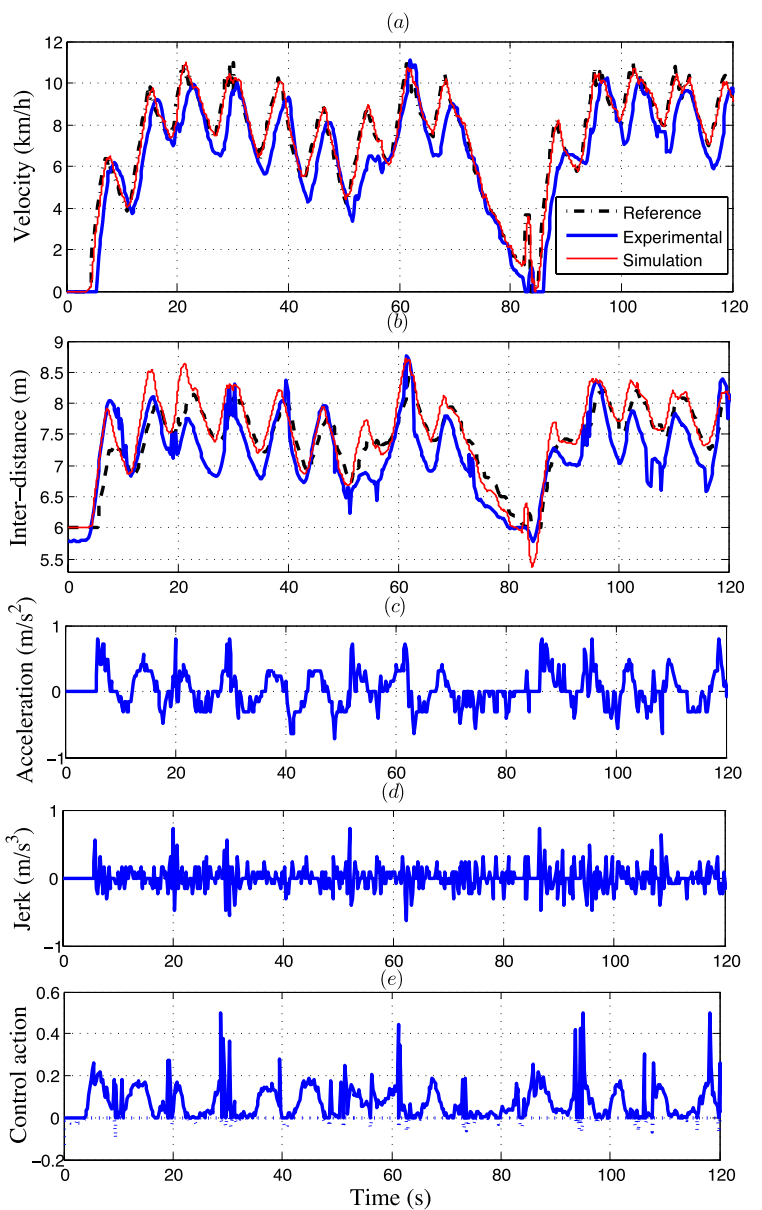

Fig. 6. ACC results using (18) as the reference interdistance $\left(d_{c}+l_{v}=6 \mathrm{~m}\right)$. (a) Velocity. (b) Interdistance. (c) Acceleration. (d) Jerk. (e) Normalized control action. (For the velocity, the reference corresponds to the velocity of the leading vehicle.)

In this way, the human influence in two consecutive trials was removed. The distance between vehicles at the beginning of the test was set to $6 \mathrm{~m}$. Once this distance was achieved with $1-\mathrm{cm}$ accuracy using the RTK-DGPS positioning system, the test was initiated. The interdistance dynamic models were parameterized to provide: 1) a maximum speed of $V_{\max }=$ $50 \mathrm{~km} / \mathrm{h} ; 2$ ) a maximum acceleration of $\gamma_{\max }=2 \mathrm{~m} / \mathrm{s}^{2}$; 3) a maximum jerk of $J_{\max }=5 \mathrm{~m} / \mathrm{s}^{3}$; and 4) a constant-time headway of $h=0.8 \mathrm{~s}$.

Figs. 6 and 7 show the behavior of the automatic vehicle when using the reference interdistance given by (18) - with $d_{c}+l_{v}=6 \mathrm{~m}$-and (19) for ACC maneuvers, respectively. Figs. 6 and 7(a) show the simulated-thinner red lines-and experimental-thicker blue lines-velocity of the following 

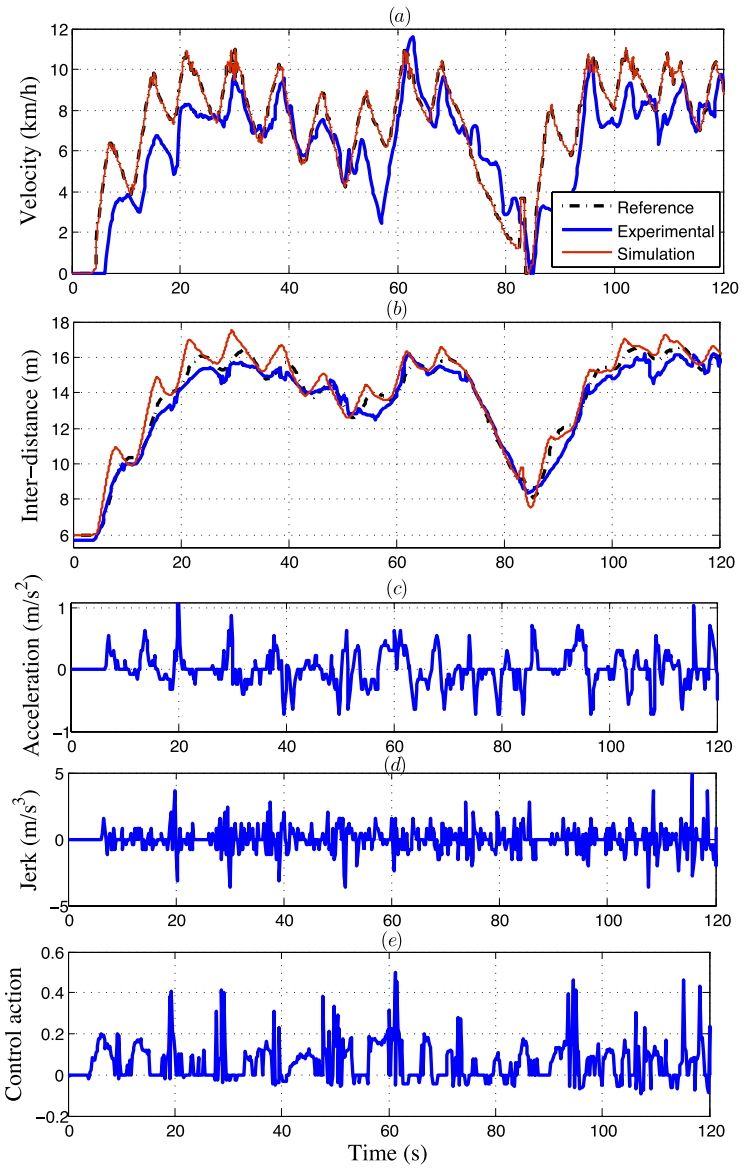

Fig. 7. ACC results using (19) as the reference interdistance. (a) Velocity. (b) Interdistance. (c) Acceleration. (d) Jerk. (e) Normalized control action (For the velocity, the reference corresponds to the velocity of the leading vehicle.)

vehicle with respect to the leading one, which is considered as reference for the former. Only slight differences can be observed between the velocity of the leader and the follower, especially in simulation. In Figs. 6 and 7(b), the desired and the experimental interdistances are represented. As observed, the actual interdistance tracks the reference interdistance adequately. Automatic vehicle's acceleration and jerk are shown in Figs. 6 and 7(c) and (d), whose values are lower than the aforementioned prerequisites. Finally, Figs. 6 and 7(e) show the normalized control action. As can be observed, the vehicle behaves efficiently during both acceleration and deceleration, even when the leading car reduces its speed significantly-at time $65 \mathrm{~s}$, the following car properly follows the reference interdistance as at the same time it increases the speed.

To compare the results for the different interdistance policies, an error function was defined as, $J=1 / T \int_{0}^{T}\left(\left|e_{p}\right|+\left|e_{v}\right|+u_{s}\right) d t$, where $e_{p}=d-d_{r}$ is the interdistance error (in $\mathrm{m}$ ), $e_{v}=V_{f_{\mathrm{ref}}}-V_{f}$ is the velocity error (in $\mathrm{km} / \mathrm{h}$ ) and $u_{s}=|d u / d t|$ is the control smoothness. Table III shows the results obtained by both strategies. As can be observed, the interdistance policy given by (19) causes smoother interdistance and, consequently, the vehicle's behavior is smoother. On the contrary, using the rule (18), the vehicle's performance is poor in comparison
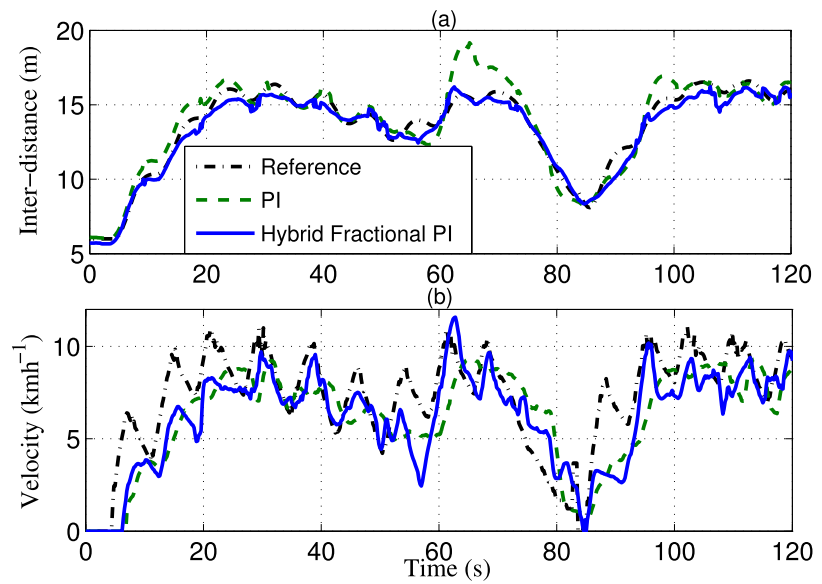

Fig. 8. Comparing ACC results of classic PI and proposed controller. (a) Interdistance (Rule (19) was used as the reference interdistance policy). (b) Velocity. (For the velocity, the reference corresponds to the velocity of the leading vehicle.)

TABLE III

ACC RESUlts WHEN USING THE REFERENCE INTERDISTANCE GIVEN BY (18) AND (19)

\begin{tabular}{|l|c|c|c|c|}
\hline Controller & $e_{p} / d_{r}$ & $e_{v}$ & $u_{s}$ & $J$ \\
\hline Inter-distance (18) & 0.049 & 1.011 & 0.216 & 1.584 \\
\hline Inter-distance (19) & 0.032 & 0.331 & 0.279 & 0.902 \\
\hline
\end{tabular}

with the previous strategy, but may be acceptable for a range of speeds.

For comparison purposes with integer-order strategies, the experimental results in [6] when applying a traditional PI controller were also considered, in which the same route was used for the tests with the interdistance policy (19) (see the interdistance and the velocity comparisons in Fig. 8). As observed, there exist meaningful differences between the hybrid fractional strategy proposed in this brief and the classical PI control, especially in terms of interdistance error and tracking, mainly due to the fact that the fractional controller allows the vehicle to follow the interdistance reference with more accuracy. On the one hand, the greatest interdistance error-with a value of $3.67 \mathrm{~m}$-is obtained with the PI controller at around $65 \mathrm{~s}$, because the autonomous vehicle is driving around a curved stretch; at that time, the error obtained with the proposed controller is almost zero. In contrast, the higher interdistance error obtained with the fractional controller is $1.54 \mathrm{~m}$ at $90 \mathrm{~s}$; at that time, the error with the PI is even greater, i.e., $1.61 \mathrm{~m}$. On the other hand, considering the mean value of the interdistance error, the proposed controller also shows a significantly better performance in ACC against the traditional PI: 0.431 against $0.658 \mathrm{~m}$, respectively. Concerning the velocity, it can be said that the PI controller follows the leading vehicle's speed with poorer results.

\section{CONCLUSION}

In this brief, a fractional-order hybrid strategy has been designed to control both the throttle and the brake pedals for $\mathrm{CC}$ and ACC maneuvers at very low speeds. Simulated and experimental results, obtained for real vehicles in a real circuit, 
were given to demonstrate the effectiveness of the proposed fractional hybrid control law.

Since the vehicle has different dynamics during accelerating and decelerating, two fractional-order PI controllers were designed for controlling the throttle and the brake for CC maneuvers. A hybrid model of the controlled system was obtained and its quadratic stability was proved using a frequency domain method, modeling the system as a switching hybrid system. ACC maneuvers were performed by two different distance policies using two cooperating vehiclesone manual, the leader, and another automatic-in which the desired interdistance between the leader and follower is maintained by an additional PD controller.

Our future effort will focus on replacing: 1) the fractional hybrid controller by a robust fractional-order PI controller to be applied to both the throttle and the brake pedals and 2) the PD interdistance controller by a fractional-order version.

\section{REFERENCES}

[1] National Center for Statistics and Analysis, "Motor vehicle traffic crash fatality counts and estimates of people injured for 2005," 2005 Annual Assessment of Motor Vehicle Crashes, NHTSA's National Center for Statistics \& Analysis, USA, Tech. Rep., 2006.

[2] D. F. Llorca et al., "Autonomous pedestrian collision avoidance using a fuzzy steering controller," IEEE Trans. Intell. Transp. Syst., vol. 12, no. 2, pp. 390-401, Jun. 2011.

[3] M. Heddebaut et al., "Millimeter-wave communicating-radars for enhanced vehicle-to-vehicle communications," Transp. Res. C, Emerging Technol., vol. 18, no. 3, pp. 440-456, Jun. 2010.

[4] V. Agarwal, N. V. Murali, and C. Chandramouli, "A cost-effective ultrasonic sensor-based driver-assistance system for congested traffic conditions," IEEE Trans. Intell. Transp. Syst., vol. 10, no. 3 , pp. 486-498, Sep. 2009.

[5] G. Naus, J. Ploeg, M. V. de Molengraft, W. Heemels, and M. Steinbuch, "Design and implementation of parameterized adaptive cruise control: An explicit model predictive control approach," Control Eng. Pract., vol. 18, no. 8, pp. 882-892, 2010.

[6] V. Milanés, J. Villagrá, J. Godoy, and C. González, "Comparing Fuzzy and intelligent PI controllers in stop-and-go manuevers," IEEE Trans. Control Syst. Technol., vol. 20, no. 3, pp. 770-778, May 2012.

[7] F. Bu and C.-Y. Chan, "English adaptive and cooperative cruise control," in English Handbook of Intelligent Vehicles. London, U.K.: Springer-Verlag, 2012, pp. 191-207.

[8] K. Lidström et al., "A modular CACC system integration and design," IEEE Trans. Intell. Transp. Syst., vol. 13, no. 3, pp. 1050-1061, Sep. 2012.

[9] R. Kianfar et al., "Design and experimental validation of a cooperative driving system in the grand cooperative driving challenge," IEEE Trans. Intell. Transp. Syst., vol. 13, no. 3, pp. 994-1007, Sep. 2012.

[10] C. A. Monje, Y. Q. Chen, B. M. Vinagre, D. Xue, and V. Feliu, Fractional-Order Systems and Controls. Fundamentals and Applications. Berlin, Germany: Springer-Verlag, 2010.

[11] I. Podlubny, "Fractional-order systems and $\mathrm{PI}^{\lambda} \mathrm{D}^{\mu}$ controllers," IEEE Trans. Autom. Control, vol. 44, no. 1, pp. 208-214, Jan. 1999.

[12] J. P. Hespanha, Encyclopedia of Life Support Systems (EOLSS). Oxford, U.K.: Eolss Publishers, 2004.

[13] N. McClamroch and I. Kolmanobsky, "Performance benefits of hybrid control design for linear and nonlinear systems," Proc. IEEE, vol. 88, no. 7, pp. 1083-1096, Jul. 2000.
[14] R. Fierro and F. Lewis, "A framework for hybrid control design," IEEE Trans. Syst., Man Cybern., A, Syst. Humans, vol. 27, no. 6, pp. 765-773, Nov. 1997.

[15] J. Lygeros, D. Godbole, and S. Sastry, "Verified hybrid controllers for automated vehicles," IEEE Trans. Autom. Control, vol. 43, no. 4 , pp. 522-539, Apr. 1998.

[16] E. Frazzoli, "Robust hybrid control for autonomous vehicle motion planning," Ph.D. dissertation, Dept. Aeronautics Astronautics, Massachusetts Inst. Technol., Cambridge, MA, USA, 2001.

[17] E. Frazzoli, M. A. Dahleh, and E. Feron, "A maneuver-based hybrid control architecture for autonomous vehicle motion planning," in SoftwareEnabled Control: Information Technology for Dynamical Systems. New York, NY, USA: Wiley, 2009, pp. 299-323.

[18] M. Mukai, T. Azuma, and M. Fujita, "A collision avoidance control for multi-vehicle using PWA/MLD hybrid system representation," in Proc. IEEE Int. Conf. Control Appl., vol. 2. Sep. 2004, pp. 872-877.

[19] C. Altafini, A. Speranzon, and K. Johansson, Hybrid Systems: Computation and Control (Lecture Notes in Computer Science). Berlin, Germany: Springer-Verlag, 2002, pp. 439-469.

[20] A. Balluchi, P. Soueres, and A. Bicchi, "Hybrid feedback control for path tracking with a bounded-curvature vehicle," in Proc. 4th Int. Workshop Hybrid Syst., Comput. Control, 2001, pp. 133-146.

[21] I. Tejado, V. Milanés, J. Villagrá, and B. M. Vinagre, "Fractional network-based control for vehicle speed adaptation via vehicle-toinfrastructure communications," IEEE Trans. Control Syst. Technol., vol. 21, no. 3, pp. 780-790, May 2013.

[22] E. Onieva, V. Milanés, C. González, T. de Pedro, J. Pérez, and J. Alonso, "Throttle and brake pedals automation for populated areas robotica," Robotica, vol. 28, no. 4, pp. 509-516, 2010.

[23] V. Milanés, D. F. Llorca, B. M. Vinagre, C. González, and M. A. Sotelo, "Clavileño: Evolution of an autonomous car," in Proc. 13th Int. IEEE Conf. Intell. Transp. Syst., Sep. 2010, pp. 1129-1134.

[24] V. Milanés, C. González, J. Naranjo, E. Onieva, and T. D. Pedro, "Electro-hydraulic braking system for autonomous vehicles," Int. J. Autom. Technol., vol. 1, no. 11, pp. 89-95, 2010.

[25] A. Rodriguez, "Estimacion de posicion y control de vehiculos autonomos a elevada velocidad," Ph.D. dissertation, Dept. Phys., Univ. Sevilla, Sevilla, Spain, 2007.

[26] A. Kamga and A. Rachid, "Speed, steering angle and path tracking controls for a tricycle robot," in Proc. IEEE Int. Symp. Comput. Aided Control Syst. Des., Sep. 1996, pp. 56-61.

[27] I. Tejado, V. Milanés, J. Villagrá, J. Godoy, H. HosseinNia, and B. M. Vinagre, "Low speed control of an autonomous vehicle by using a fractional PI controller," in Proc. 18th IFAC World Congr., vol. 18, 2011, pp. 15025-15030.

[28] S. H. HosseinNia, I. Tejado, B. M. Vinagre, V. Milanés, and J. Villagrá, "Low speed control of an autonomous vehicle using a hybrid fractional order controller," in Proc. 2nd ICCIA, 2011, pp. 1-12.

[29] J. Villagrá, V. Milanés, J. Pérez, and T. de Pedro, "Control basado en PID inteligentes: Aplicación al control de crucero de un vehículo a bajas velocidades," Rev. Iberoamer. de Autom. Inf. Ind., vol. 7, no. 4, pp. 44-52, 2010.

[30] C. A. Monje, B. M. Vinagre, Y. Q. Chen, and V. Feliu, "On fractional $\mathrm{PI}^{\lambda}$ controllers: Some tuning rules for robustness to plant uncertainties," Nonlinear Dyn., vol. 38, nos. 1-4, pp. 369-381, 2004.

[31] S. H. HosseinNia, I. Tejado, and B. M. Vinagre, "Stability of fractional order switching systems," Comput., Math. Appl., vol. 66, no. 5, pp. 585-596, 2013.

[32] J. Zhou and H. Peng, "Range policy of adaptive cruise control vehicles for improved flow stability and string stability," IEEE Trans. Int. Transp. Syst., vol. 6, no. 2, pp. 229-237, Jun. 2005.

[33] J. Martinez and C. C. de Wi, "A safe longitudinal control for adaptive cruise control and stop-and-go scenarios," IEEE Trans. Control Syst. Technol., vol. 15, no. 2, pp. 246-258, Mar. 2007.

[34] P. A. Cook, "Stable control of vehicle convoys for safety and comfort," IEEE Trans. Autom. Control, vol. 52, no. 3, pp. 526-531, Mar. 2007. 